\title{
Coexistence of EGFR with KRAS, or BRAF, or PIK3CA somatic mutations in lung cancer: a comprehensive mutation profiling from 5125 Chinese cohorts
}

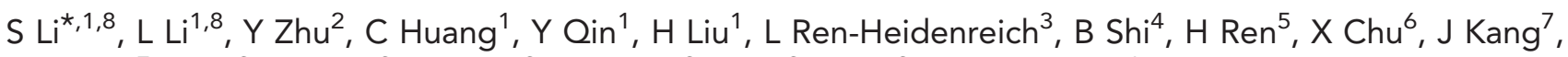

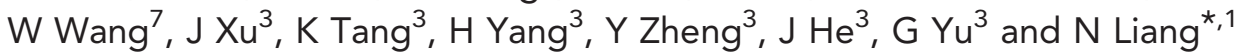

${ }^{1}$ Department of Thoracic Surgery, Peking Union Medical College Hospital, Beijing 100730, China; ${ }^{2}$ Department of Thoracic Surgery, Air Force General Hospital of PLA, Beijing 100036, China; ${ }^{3}$ SurExam Bio-Tech Co., Guangzhou 510663, China; ${ }^{4}$ Department of Thoracic Surgery, China-Japan Friendship Hospital, Beijing 100029, China; ${ }^{5}$ Department of Thoracic Surgery, General Hospital of Armed Police Forces, Beijing 100039, China; ${ }^{6}$ Department of Thoracic Surgery, Chinese PLA General Hospital, Beijing 100853, China and ${ }^{7}$ China Navy General Hospital, Beijing 100037, China

Background: Determining the somatic mutations of epidermal growth factor receptor (EGFR)-pathway networks is the key to effective treatment for non-small cell lung cancer (NSCLC) with tyrosine kinase inhibitors (TKIs). The somatic mutation frequencies and their association with gender, smoking history and histology was analysed and reported in this study.

Methods: Five thousand one hundred and twenty-five NSCLC patients' pathology samples were collected, and EGFR, KRAS, BRAF and PIK3CA mutations were detected by multiplex testing. The mutation status of EGFR, KRAS, BRAF and PIK3CA and their association with gender, age, smoking history and histological type were evaluated by appropriate statistical analysis.

Results: EGFR, KRAS, BRAF and PIK3CA mutation rates revealed $36.2 \%, 8.4 \%, 0.5 \%$ and $3.3 \%$, respectively, across the 5125 pathology samples. For the first time, evidence of KRAS mutations were detected in two female, non-smoking patients, age 5 and 14, with NSCLC. Furthermore, we identified 153 double and coexisting mutations and 7 triple mutations. Interestingly, the second drug-resistant mutations, $\mathrm{T} 790 \mathrm{M}$ or E545K, were found in 44 samples from patients who had never received TKI treatments.

Conclusions: EGFR exons 19, 20 and 21, and BRAF mutations tend to happen in females and non-smokers, whereas KRAS mutations were more inclined to males and smokers. Activating and resistant mutations to EGFR-TKI drugs can coexist and 'second drug-resistant mutations', T790M or E545K, may be primary mutations in some patients. These results will help oncologists to decide candidates for mutation testing and EGFR-TKI treatment.

Lung cancer has the highest incidence among all cancers and is the leading cause of death worldwide (Jemal et al, 2011). Non-small cell lung cancer (NSCLC) accounts for the vast majority of lung cancers $(\sim 80 \%)$ (American Cancer Society, 2011). Treating advanced NSCLC with the small molecule tyrosine kinase inhibitors (TKIs), gefitinib and erlotinib, which target the epidermal growth factor receptor (EGFR), has significantly improved the overall survival rate of patients harbouring activating mutations (Pao et al, 2005; Toyooka et al, 2005).

This report serves to provide oncologists with patient demographics associated with a higher statistical probability of having somatic mutations of the EGFR signalling pathway networks.

\footnotetext{
*Correspondence: Dr N Liang; E-mail: happinessliang80@sina.com or Professor S Li; E-mail: doctorlishanqing@sina.com

${ }^{8}$ These authors contributed equally to this work
}

Received 20 November 2013; revised 14 March 2014; accepted 22 March 2014; published online 17 April 2014

(C) 2014 Cancer Research UK. All rights reserved 0007 - 0920/14 
Owing to the large cohort of patients, we were able to analyse the coexistence of double or triple somatic mutations of the relevant genes. Although they are rare, multiple mutations are very critical for the decision of TKI treatment. Importantly, for the first time, we report here detection of KRAS somatic mutations in NSCLC samples obtained from non-smoking children, which may be associated with second-hand smoke exposure or some environmental factors.

EGFR, KRAS, BRAF or PIK3CA mutations have been shown to predict clinical response to EGFR-TKIs in NSCLC patients. Mutations of these four genes are associated with gender, smoking history and histology. For example, deletions in EGFR exon 19 and the point mutation L858R in exon 21 are the most common activating mutations and have been predominantly found in females, never smokers, adenocarcinomas and Asian patients (Rosell et al, 2009; Xu et al, 2011). In addition, they have been identified as predictors of responsiveness to both gefitinib and erlotinib (Lynch et al, 2004; Pao et al, 2004). KRAS, BRAF or PIK3CA mutations are also important indicators for EGFR-TKI therapy (Marchetti et al, 2005; Pao et al, 2005; Schmid et al, 2009; Ludovini et al, 2011; Paik et al, 2011; Dogan et al, 2012). Conversely, KRAS mutations are more common in individuals with a history of cigarette use and are associated with resistance to EGFR-TKI (Pao et al, 2005; Xu et al, 2011; Dogan et al, 2012). KRAS, mutated to an oncogenic form by introducing amino-acid substitutions at codons 12,13 and 61 , are detected in $25-33 \%$ of patients with lung adenocarcinomas from the United States and Europe (Smits et al, 2012) and 8\% from China (Xu et al, 2011). BRAF, a serine/threonine kinase, is activated by somatic point mutation $\mathrm{V} 600 \mathrm{E}$ in exon 15 in only $3 \%$ of lung cancer patients. $B R A F$ mutations are associated with resistance to TKI therapy (Pao et al, 2005; Lin and Bivona, 2012). PIK3CA encodes the p110 $\alpha$ subunit of the mitogenic signalling protein phosphatidylinositol 3-kinase (PI3K). PIK3CA mutations in the helical-binding domain and the catalytic subunit of the protein have been associated with tumourigenesis and treatment resistance in various malignancies. Indeed, PIK3CA mutations are detected in $\sim 4 \%$ of lung cancers and have become an important predictor for drug resistance to EGFR-TKI (Ludovini et al, 2011).

Owing to very low frequencies, the clinical significance of coexistence of somatic mutations has never been systematically analysed up until now. Although coexistence of somatic mutations on EGFR pathway networks have been observed in some individual cases (Toyooka et al, 2005), the low frequency of this occurrence has presented limitations to determining the clinical implications for these multiple mutation cases. Accordingly, large-scale mutation analysis could identify the frequencies of multiple mutations and expand our understanding of somatic mutations on the EGFR pathway networks.

Using a liquid chip technology for somatic mutation identification (Wu et al, 2010; Li et al, 2011), we conducted a large-scale mutation analysis for EGFR, KRAS, BRAF, or PIK3CA mutations on 5125 tumour samples from patients with NSCLC, and analysed their associations with gender, smoking and histology. Of these, 160 cases were identified as having multiple mutations. In this study, the clinical significance of these 160 cases has been analysed and is discussed.

\section{MATERIALS AND METHODS}

Patients. Between 2009 and 2012, 5125 patients with lung cancer from most major hospitals throughout China were enrolled in this study. Formalin-fixed and paraffin-embedded (FFPE) tumour samples were prepared from primary surgical or biopsy specimens in lung. All samples were identified by pathologists as primary
NSCLC and were provided by the SurExam Clinical Testing Centre. Written informed consent was obtained from all participants.

Mutation analysis of EGFR, KRAS, BRAF and PIK3CA. Tumour genomic DNA from each FFPE slide was extracted with the Maxwell system (Promega, Madison, WI, USA). The mutation status was analysed with the 70plex liquidchip platform (Surexam, Guangzhou, China) for the 70 alleles (Li et al, 2011). The 70plex includes five major steps: (1) multiplex PCR to amplify 70 target genes; (2) exonuclease I and shrimp alkaline phosphatase (EXO$\mathrm{SAP}$ ) cleaning to remove excess nucleotides and primers; (3) allelespecific primer extension where EXO-SAP-cleaned PCR products were amplified with70 specific primers that were linked to 70 universal tags; (4) hybridisation to beads and (5) Luminex analysis and the median fluorescence intensity was read and analysed. The 70plex platform has been validated for clinical use. All of 5125 tests and data analyses were performed at the SurExam Clinical Testing Centre. As part of quality control, samples were randomly sent to independent companies for DNA sequencing analysis.

Statistical analysis. The mutation status of EGFR, KRAS, BRAF and PIK3CA and their association with gender, age and smoking history were evaluated using Maximum Likelihood Multivariate Logistic Regression. Variables were selected by the Complete Model. The adjusted odds ratios were calculated. A two-sided $P$-value of $<0.05$ was considered statistically significant if there was no alpha correction. The phenotypes of gender and smoking were analysed using the Binomial test and then the Bonferroni correction was performed. Smoking refers to current and ever smoking in this study. The significant difference refers to $P<0.001$ between smoking and non-smoking or male and female proportions in comparison with overall samples and phenotypes. All analyses were performed using SAS 9.2 software (SAS Institute Inc., Cary, NC, USA).

\section{RESULTS}

Demographic characteristics. The presence of EGFR, KRAS, $B R A F$ and PIK3CA mutations was analysed in 5125 lung cancer patients; 2072 of them were female (40.4\%) and 3053 male (59.6\%). Patient ages ranged from 5-91 years with the median age of 59 years. All specimens were NSCLC. Non-small cell lung cancer forms were identified in all of patients: 4046 (78.9\%) samples were adenocarcinomas, whereas only 1079 (21.1\%) were squamous cell carcinomas (see Table 1).

Mutation distributions. Two thousand two hundred and eight (2208) NSCLC patients (2208 out of 5125, 43.1\%) harboured single mutations of EGFR, KRAS, BRAF or PIK3CA, and 160 were found to have multiple mutations (Figure 1, 153 double and coexisting mutations and seven triple mutations). Of the 153 double and coexisting mutations, 58 patients carried EGFR + PIK3CA mutations; 53, two EGFR double mutations; 29, EGFR + KRAS mutations; 9, KRAS + PIK3CA mutations; 2, EGFR + BRAF mutations; and 2, BRAF + PIK3CA mutations. Of the seven triple mutations, five patients carried $2 \times E G F R+1 \times P I K 3 C A$ mutations; one patient carried $1 \times E G F R+2 \times$ PIK3CA mutations; and one patient carried $1 \times E G F R+1 \times K R A S+1 \times P I K 3 C A$ mutations (Figure 1B).

Together, there were $36.2 \%$ patients with EGFR mutations (1854 out of 5125); 8.4\%, KRAS mutations (429 out of 5125); 0.5\%, BRAF mutations (26 out of 5125) and 3.3\%, PIK3CA mutations (167 out of 5125). The percentage distributions of EGFR, KRAS, BRAF and PIK3CA among mutation-positive samples were $74.9 \%, 17.3 \%$, $1.1 \%$ and $6.7 \%$, respectively (Figure $2 \mathrm{~B}$ ). 


\begin{tabular}{|c|c|}
\hline & No. of patients \\
\hline Median age & $59(5-91)$ years \\
\hline \multicolumn{2}{|l|}{ Gender } \\
\hline Male & 3053 \\
\hline Female & 2072 \\
\hline \multicolumn{2}{|l|}{ Histology ${ }^{a}$} \\
\hline ADCs & 4046 \\
\hline Squamous & 1079 \\
\hline \multicolumn{2}{|c|}{ Smoking history } \\
\hline Smokers ${ }^{\mathbf{b}}$ & 2314 \\
\hline Non-smokers ${ }^{c}$ & 2493 \\
\hline Unknown & 318 \\
\hline \multicolumn{2}{|c|}{ 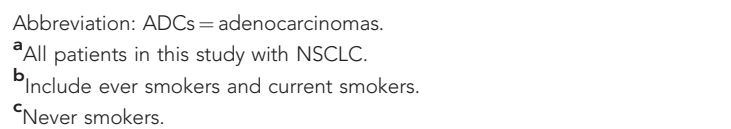 } \\
\hline
\end{tabular}

EGFR mutations. EGFR mutations were detected in $36.2 \%$ patients (1854 out of 5125), with most of these located in exon $19(18.0 \%, 920$ out of 5125$)$ or exon $21(16.9 \%, 868$ out of 5125$)$. The remaining of these were located in exon $20(1.5 \%, 75$ out of $5125)$ or exon $18(1.0 \%, 49$ out of 5125). The percentage distributions in exons 18,19, 20 and 21 were 3\%, 48\%, 4\% and $45 \%$, respectively, (Figure 2B). Double EGFR mutations were found in $1.1 \%$ patients (58 out of 5125 ) (Figure $2 \mathrm{~A}$ ).

KRAS mutation. KRAS mutation was detected in $8.4 \%$ patients (429 out of 5125), with most of these located in exon 2 (8.0\%, 408 out of 5125); the remaining ones were detected in exon 3 $(0.4 \%, 21$ out of 5125$)$ (Figure 2). In these KRAS mutation specimens, there were $134 \mathrm{G} 12 \mathrm{C}$ mutations and $87 \mathrm{G} 12 \mathrm{~V}$ mutations (Figure 2). Notably, there were two patients under or near the age of 15 years, who were found to carry the KRAS mutation. The youngest of these patients, who was 5 years of age, suffered from NSCLC and was also found to carry a KRAS E3 mutation $(\mathrm{Q} 61 \mathrm{H})$. The other of these patients, who was 14 years of age, had a KRAS E2 mutation $(\mathrm{G} 12 \mathrm{~V})$. Interestingly, neither of these two patients was a smoker, and both were female.

BRAF mutation. BRAF mutation was detected in $0.5 \%$ patients (26 out of 5125). All of them were a V600E mutation (Figure 2).

PIK3CA mutation. PIK3CA mutation was detected in $3.3 \%$ patients (167 out of 5125 ) with $2.1 \%$ of these mutations located in exon 9 (109 out of 5125) and $1.1 \%$ in exon 20 (58 out of 5125) (Figure 2). There were 62 cases of E545K mutations and 44 cases of E542K (Figure 2). We found one patient who carried two PIK3CA mutations plus one EGFR mutation (E545K + E542K + L861Q) (Figure 1B).

Patients' characteristics and somatic mutations. Analysis by multivariate logistic regression revealed that females were more likely to have E19/E21 mutations (male vs female: $23.5 \%$ vs $51.5 \%$, adjusted OR 0.63 , CI $0.52-0.75, P \leqslant 0.001$ ) than males (Table 2). Similar results were also found in $B R A F$ mutations (male $v s$ female: $0.2 \%$ vs $1.1 \%$, adjusted OR 0.15 , CI $0.04-0.62, P \leqslant 0.01$ ). The KRAS mutation rate was higher in males than in females (male $v s$ female: $11.0 \%$ vs $6.1 \%$, adjusted OR 1.38 , CI $0.99-1.93, P=0.054$ ), but the difference was not statistically significant. These results suggested a significant association of EGFR E19/E21 and BRAF mutations with gender.

Non-smokers were more likely to have E19/E21 mutations than smokers (smokers vs non-smokers: $18.1 \%$ vs 51.0\%, adjusted OR 0.287, CI 0.24-0.34, $P \leqslant 0.001$ ) and E20 mutations (smokers vs non-smokers: $0.6 \%$ vs $2.5 \%$, adjusted OR 0.39 , CI $0.17-0.87$, $P=0.022)$. However, an inverse relationship was found in $K R A S$ mutations (smokers vs non-smokers: $6.0 \%$ vs $12.3 \%$, adjusted OR 1.83 , CI $1.38-2.57, P \leqslant 0.001$ ). There was no significant difference between smoker and non-smoker NSCLC patients for the mutation rates of EGFR E18, BRAF and PIK3CA.

EGFR E19/E21 (adeno vs squamous: $42.0 \%$ vs $8.2 \%$, adjusted OR 7.36, CI 5.61-9.57, $P \leqslant 0.001$ ), EGFR E20 (adeno vs squamous: $1.9 \%$ vs $0.5 \%$, adjusted OR 2.89 , CI $1.04-8.04, P=0.042)$ and $K R A S$ (adeno vs squamous: $10.4 \%$ vs $3.6 \%$, adjusted OR 3.26, CI $2.21-$ $4.80, P \leqslant 0.001)$ mutations were more frequent in adenocarcinomas. However, the PIK3CA mutation rate in adenocarcinomas was lower than in squamous cell carcinoma (adeno $v s$ squamous: $2.9 \%$ vs $7.9 \%$, adjusted OR 0.50 , CI $0.34-0.73, P \leqslant 0.001$ ). These results suggest a significant association of EGFR E19/E21, EGFR E20, KRAS and PIK3CA mutations with the histology of tumour specimens. Accordingly, adenocarcinomas have a higher likelihood of harbouring EGFR E19/E21, EGFR E20 and KRAS mutations, whereas squamous cell carcinomas have a higher likelihood of harbouring a PIK3CA mutation. Unfortunately, there was no classification information on 3948 NSCLC samples (out of 5125) at time of testing.

In addition, there was no significant difference found for the mutation rates of EGFR, KRAS, BRAF and PIK3CA occurring in between patients over 60 years of age and those 60 years of age or younger. We also conducted statistical analysis by decade of age; there was no significant difference of these four gene mutations in any of the age groups (data not shown).

We conclude, therefore, that non-smoking females with adenocarcinoma lung cancer may have a high likelihood of carrying the EGFR E19/E21 mutation, whereas smokers with adenocarcinoma lung cancer have a high likelihood of carrying KRAS mutations.

Phenotype analysis with smoking history and gender. A significant association was found between smoking history and EGFR pathway gene mutation frequencies (Figure 3): the proportions of smoking vs non-smoking in E746_A750del, L747_A750 > P, L747_T751del, L747_P753 > S, T790M，L858R and $\mathrm{G} 12 \mathrm{C}$ mutations were significantly different with the proportion of smoking $v s$ non-smoking in the overall sample $(P \leqslant 0.001)$. Never smokers showed higher in E746_A750del, L747_A750 > P, L747_T751del, L747_P753 > S, T790M and L858 $\overline{\mathrm{R}}$ mutations. However, there was an inverse relationship in G12C mutations. These results suggest a significant association of mutation phenotypes with smoking history.

Significant associations were also found between gender and mutation phenotypes. The proportions of male vs female in E746_A750del, L747_P753 > S, T790M, L858R, L861Q, V600E and G12C mutations were significantly different with the proportion of male $v s$ female in the overall sample $(P \leqslant 0.001)$. E746_A750del, L747_P753 > S, T790M, L858R, L861Q and V600E mutations were higher in females than males, whereas G12C was higher in males than in females (Figure 3).

Double mutations. There were 153 patients found to have a double and coexisting mutation profile; $72.5 \%$ of them (111 out of 153) were never smokers; 62.1\% (95 out of 153) were females (Figure 1A). Interestingly, there were only $13.1 \%$ (20 out of 153) of these who were treated with the TKI or TKI-combined therapy before the tests. 


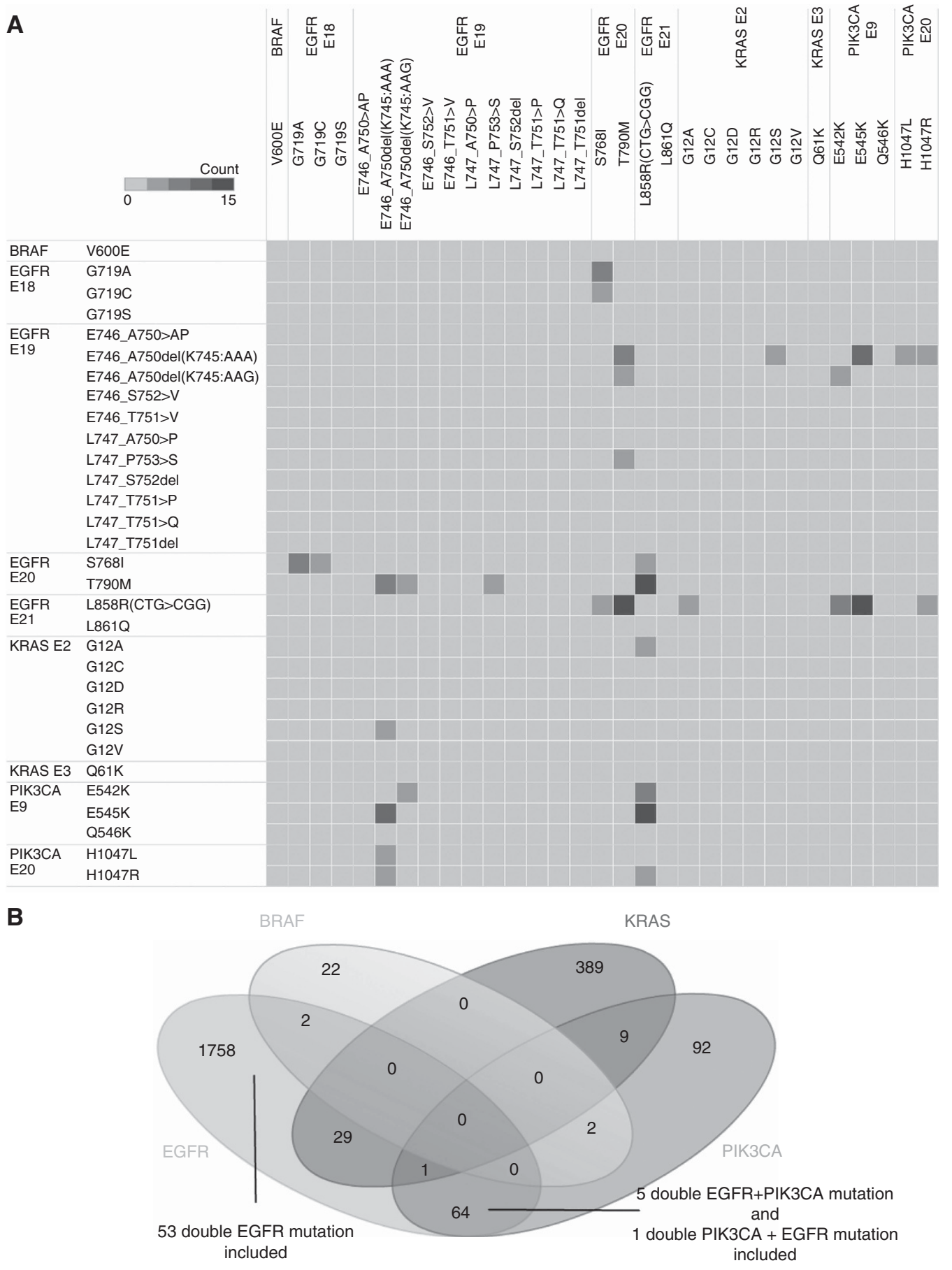

Figure 1. Combinations of multiple mutations. (A) Double mutation sites and case number in 153 patients. Double mutations L858R $+\mathrm{T790M}$ showed the highest incidence rate (9.8\%, 15 out of 153) followed by L858R + E545K (8.5\%, 13 out of 153). (B) Four set venn-diagram of single and multiple mutation panoramagram for the whole study.

There were 30 EGFR mutation specimens that also harboured KRAS mutations; two had BRAF mutations; and 65 had PIK3CA mutations. There were $6.0 \%$ (10 out of 167 ) of KRAS mutation specimens that also harboured PIK3CA mutations. However, KRAS and $B R A F$ mutations were mutually exclusive.

Double mutations L858R and T790M possessed the highest incidence rate $(9.8 \%, 15$ out of 153) followed by L858R + E545K $(8.5 \%, 13$ out of 153$)$ (Figure 1A).

It is well known that the EGFR E19/E21 mutation is a sensitive or active mutation to TKI therapy, whereas T790M and E545K are resistant mutations. We identified 56 patients who carried both E19/E21 mutations and T790M or E545K; most of these were female $(73.2 \%, 41$ out of 56$)$ and never smokers (78.6\%, 44 out of
56). Of these, $53.6 \%$ (30 out of 56) were detected in patients $<60$ years of age, and $78.6 \%$ (44 out of 56) had never received TKI treatments (data not shown).

Triple mutations. There were seven patients found to carry a triple mutation: four of them were male; two, smokers; age range, 47-76 years. There were five patients who harboured double EGFR mutations and one patient (76 years old, female) who had double PIK3CA mutations. Three triple-mutation specimens harboured EGFR E21, and all EGFR E21 mutations in this triplet mutation group were L861Q. The samples with double or triple mutations have been further confirmed by independent DNA sequencing lab (data not shown). 
A

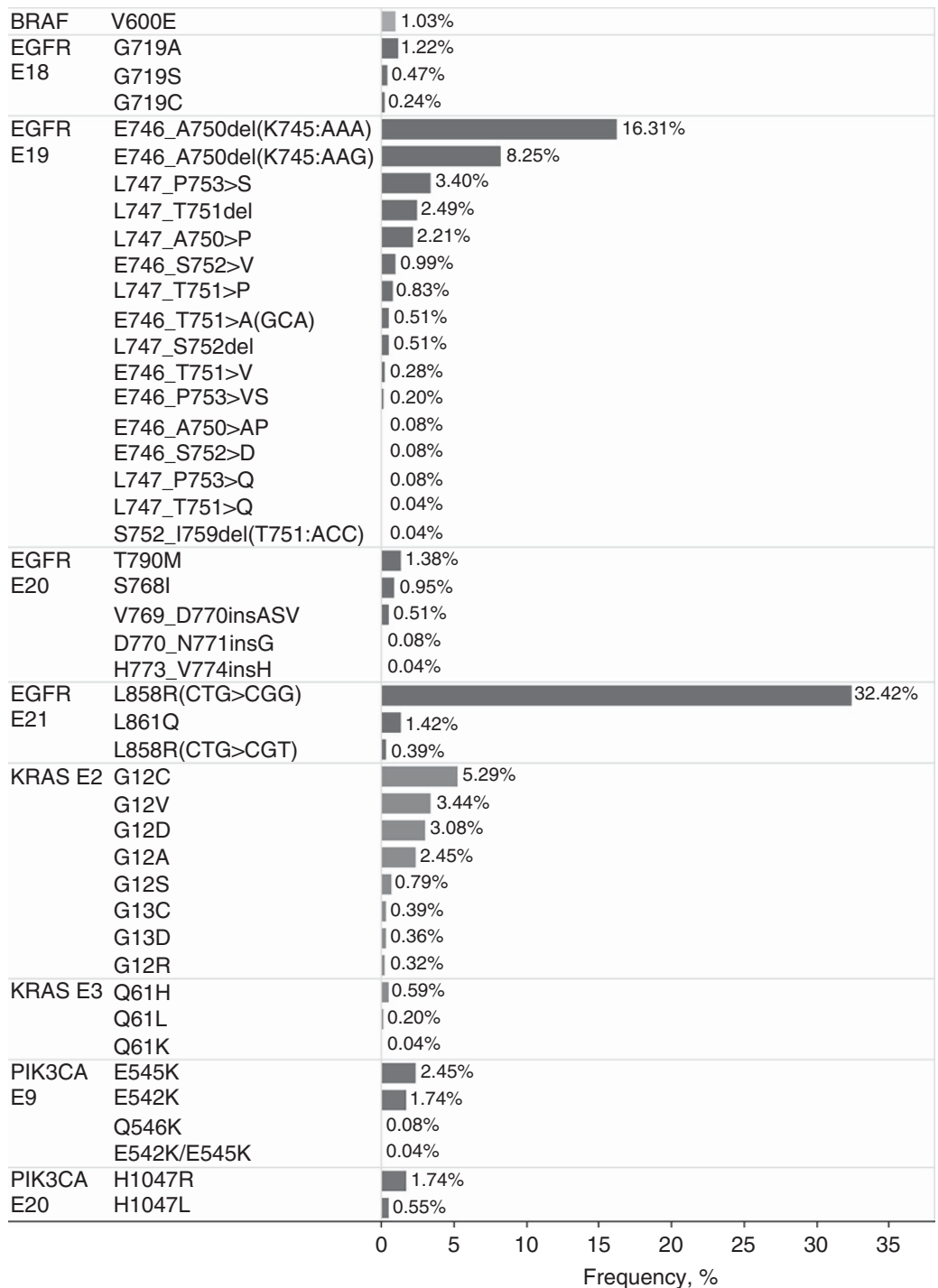

B

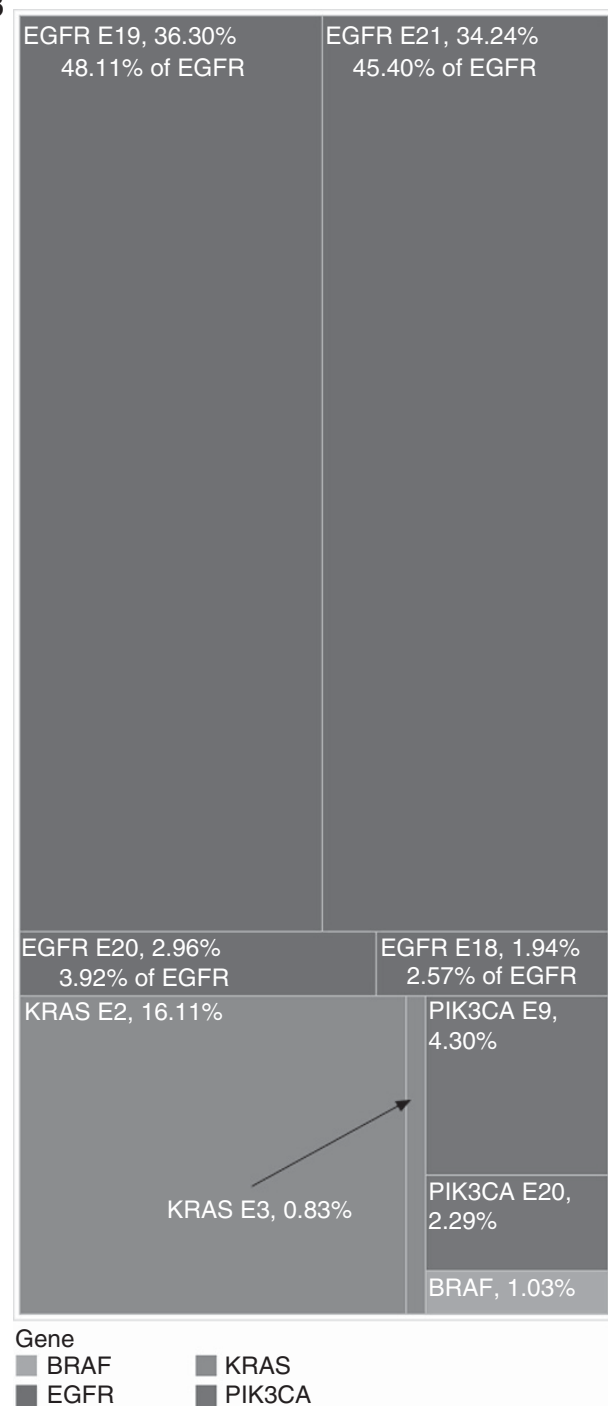

Figure 2. Somatic mutation frequencies of EGFR, KRAS, BRAF and PIK3CA. (A) The mutation sites (abscissas) and frequency (ordinates) of EGFR, KRAS, BRAF and PIK3CA in 5125 patients with NSCLC. (B) Distributions tree-map of EGFR, KRAS, BRAF and PIK3CA mutations in 2368 patients carrying mutations.

Table 2. Multivariate logistic regression of objective gene mutations

\section{Covariates for logistic regression}

\begin{tabular}{|c|c|c|c|c|c|c|c|c|}
\hline \multirow[b]{4}{*}{ Gene mutation } & \multicolumn{8}{|c|}{ Covariates for logistic regression } \\
\hline & \multicolumn{2}{|l|}{ Gender } & \multicolumn{2}{|l|}{ Age } & \multicolumn{2}{|c|}{ Smoking } & \multicolumn{2}{|c|}{ Histology subtype } \\
\hline & \multicolumn{2}{|c|}{ Male vs female ${ }^{a}$} & \multicolumn{2}{|c|}{$>60$ vs $<60^{a}$} & \multicolumn{2}{|c|}{ Smokers vs never smokers ${ }^{a}$} & \multicolumn{2}{|c|}{ ADCs vs SCCs ${ }^{a}$} \\
\hline & AOR $(95 \% \mathrm{Cl})$ & $P$-value & AOR $(95 \% \mathrm{Cl})$ & $P$-value & AOR $(95 \% \mathrm{Cl})$ & $P$-value & AOR $(95 \% \mathrm{Cl})$ & $P$-value \\
\hline EGFR E19/E21 & $0.628(0.524-0.754)$ & $<0.0001$ & $1.040(0.898-1.204)$ & 0.6002 & $0.287(0.237-0.347)$ & $<0.0001$ & $7.358(5.611-9.566)$ & $<0.0001$ \\
\hline EGFR E18 & $0.501(0.222-1.132)$ & 0.0964 & 1.509 (0.813-2.799) & 0.1922 & $0.676(0.284-1.608)$ & 0.3762 & $1.617(0.627-4.169)$ & 0.3200 \\
\hline EGFR E20 & $0.540(0.273-1.070)$ & 0.0775 & $0.889(0.532-1.483)$ & 0.6513 & $0.387(0.173-0.870)$ & 0.0216 & $2.889(1.039-8.035)$ & 0.0420 \\
\hline KRAS & $1.384(0.995-1.926)$ & 0.0537 & $1.173(0.933-1.476)$ & 0.1719 & $1.833(1.380-2.568)$ & $<0.0001$ & $3.256(2.209-4.798)$ & $<0.0001$ \\
\hline BRAF & $0.147(0.035-0.623)$ & 0.0093 & $0.642(0.251-1.646)$ & 0.3567 & $1.425(0.371-5.476)$ & 0.6057 & $1.053(0.302-3.675)$ & 0.9352 \\
\hline PIK3CA & $1.093(0.666-1.795)$ & 0.7248 & $0.840(0.582-1.212)$ & 0.3512 & $1.099(0.682-1.772)$ & 0.6978 & $0.495(0.335-0.733)$ & 0.0004 \\
\hline
\end{tabular}



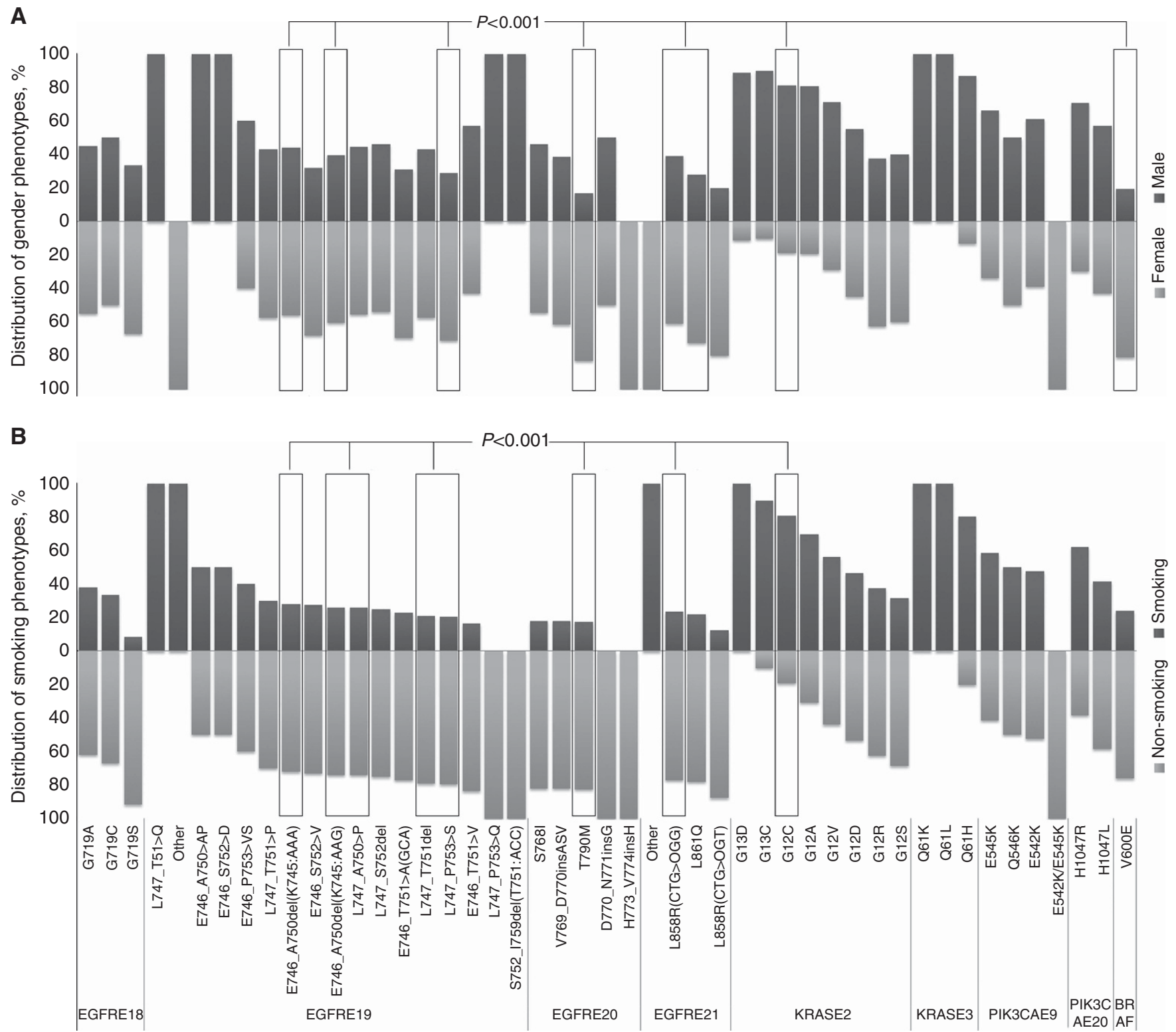

Figure 3. (A) The bars on two sides of the horizontal axis represent the percentage difference of male and female genotypes. The bars above represent male genotypes, and the bars below represent female genotypes. The bars with a rectangle box show the statistical difference of genotypes between male and female by the binomial test $(P<0.001)$. (B) The bars on two sides of horizontal axis represent the percentage difference of smoker and non-smoker genotypes. The above are smokers', and the below, non-smokers'. The bars with a rectangle box show the statistical difference of genotypes in percentage between smokers and non-smokers by the binomial test $(P<0.001)$. Chart $(\mathbf{A})$ and $(\mathbf{B})$ share the same horizontal axis that represents genotypes.

\section{DISCUSSION}

We tested 5125 Chinese NSCLC specimens for the somatic mutation frequencies of EGFR, KRAS, BRAF and PIK3CA. It was expected that somatic mutation testing from such a large cohort of NSCLC patients would provide more reliable results for interpreting the association of mutation frequencies with patient demographics. In this study, we identified 34.8\% EGFR E19/E21, 2.2\% EGFR E18/E20, 8.4\% KRAS, 0.5\% BRAF and 3.3\% PIK3CA mutations within the population (Figure 2B). Age had no significant role in mutations of these four genes. Although most of the EGFR mutations were detected in adenocarcinomas, from the unselected Chinese patients with NSCLC, the EGFR E19/E21 mutation frequencies were much higher than previously reported in American patients with adenocarcinoma NSCLC (34.8\% from
5125 patients $v$ s $19 \%$ from 2142 patients) suggesting that racial diversity contributes to occurrence of the E19/E21 mutation (D’Angelo et al, 2011). We also observed that EGFR E19/E21 mutations were significantly higher in NSCLC from tumours with adenocarcinoma histology ( $43.6 \%$ vs $8.5 \%, P \leqslant 0.001$ ), in women (51.5\% vs $23.5 \%, P \leqslant 0.001)$ and in never smokers (51.0\% vs $18.1 \%$, $P \leqslant 0.001$ ), which are similar to results of previous reports (Xu et al, 2011; Dogan et al, 2012).

From our 1854 EGFR-mutated cases, mutations in exons 18, 19, 20 and 21 occurred in 3\%,48\%, 4\% and 45\%, respectively. These findings are consistent with the frequencies of these mutations reported for an international database by researchers from the Metropolitan Hospital of Athens who collected data reported on 3303 EGFR mutations from peer-reviewed publications and concluded that the mutation distribution among these four exons are $4 \%, 50 \%, 6 \%$ and $40 \%$, respectively (Somatic Mutation in 
Epidermal Growth Factor Receptor Database http://www.somaticmutations-egfr.info/overall_mutation_frequency.html).

Although the EGFR exon 20 S768I mutation is considered to be a very rare mutation, we detected a total of $24(0.5 \%)$ NSCLC harbouring this mutation. Interestingly, we found high frequencies of S768I in multiple mutation cases: in two triple-mutation cases ( 2 out of 7 ) and in 15 double-mutation cases (15 out of 153), in four cases of L858R/S768I and in 11 cases of G719X/S768I. The function of the S768I mutation in TKI therapy is still uncertain. Masago et al (2010) demonstrated that one patient who harboured the S768I mutation showed a good clinical response to EGFR-TKI therapy, whereas Asahina et al (2006) reported completely opposite results. The fact that S768I showed high frequencies in multiple mutations suggests a rationale for specific studies designed to explore the role of S768I in NSCLC oncogenesis.

In contrast to EGFR mutations, KRAS codon 12 and 13 mutations are typically found in smokers, are associated with a poor NSCLC prognosis, are resistant to TKIs and predict lack of a survival benefit from adjuvant chemotherapy. These mutations have been found to have an association with race: 25\% NSCLC in western countries and much less in Asian NSCLC (Rodenhuis et al, 1987; Slebos et al, 1990; Riely et al, 2008). In our study, only $12.3 \%$ smokers and $6.0 \%$ non-smokers were detected to harbour the KRAS mutation in the 5125 NSCLC cases, which is much lower than that previously reported in the United States (34\% in smokers and $6 \%$ in non-smokers) (Dogan et al, 2012). Initially, KRAS mutations were thought to be uncommon in NSCLC cases without a history of smoking (Rodenhuis et al, 1987). However, we identified $6.0 \%$ of KRAS mutations in non-smokers as also observed in the United States (Riely et al, 2008; Dogan et al, 2012), which may lead future studies to explore the impact of factors other than cigarettes, such as genetic, environmental or hormonal factors, on KRAS mutations.

Patients with BRAF V600E mutations in their tumours had significantly shorter DFS and OS times than those without mutations (Marchetti et al, 2011). To our knowledge, this is the largest cohort of V600E tests performed on NSCLC samples obtained from patients of the same race. The frequency of V600E is $0.51 \%$ in our study, which is much lower than that reported for Italian (2.8\%) (Marchetti et al, 2011) and American patients (2.9\%), but comparable to Japan patients (0.8\%) (Sasaki et al, 2006). Therefore, we suspect that like EGFR mutations, incidence of V600E mutations may be racial reliant. We also did not see significantly higher V600E mutations in smokers than nonsmokers. Similar results were reported by Marchetti et al (2011) as well. However, our data indicate that $\mathrm{V} 600 \mathrm{E}$ mutations are significantly more prevalent in females than in males $(P \leqslant 0.05)$.

The median age of NSCLC is 71 worldwide (Subramanian et al, 2010). However, it is 59 in our study, which is much lower than 71. Although the median age in Asia has been reported as lower than 71 before (Xu et al, 2011; Zhang et al, 2012), more young patients with NSCLC in China than the western countries may be the reason for the low median age. According to an internet database from 1381 hospitals, there is only $0.7 \%$ lung cancer at the age group $<40$ years (Age Group of Lung Cancer Diagnosed, 2000-2007). However, our patient population included 176 (3.4\%) NSCLC patients who were younger than 40 years of age. Lung cancer is extremely rare in children. Surprisingly, for the first time, we discovered KRAS mutations in two very young patients: a 5-year old (with a Q61H mutation) and a 14-year old (with a $\mathrm{G} 12 \mathrm{~V}$ ). Both of these patients were non-smoker females. This may be the first report of KRAS somatic mutation cases in children with NSCLC. Although it is a widely accepted notion that second-hand smoke (SHS) is linked to lung cancer (Besaratinia and Pfeifer, 2008), medical professionals and researchers have not reached a consensus on the extent of the increase in lung cancer risk due to SHS. Although recent data suggested that most KRAS mutations in non-smokers are not due to SHS exposure (Dogan et al, 2012), we raised the possibility again that certain subtypes of KRAS mutations in children may result from SHS. The epidemiology of young NSCLC in China and association with SHS and environmental factors are currently under investigation.

Coexistence of the PIK3CA mutation and other activating gene mutations in the EGFR signalling pathway were previously reported (Chaft et al, 2012). Double mutations of KRAS and PIK3CA exon 9 (but not exon 20) have also been reported in colorectal cancer (De Roock et al, 2010). However, in lung cancer, EGFR gene mutations are reported as mutually exclusive with $K R A S$ or BRAF gene mutations (Marchetti et al, 2005). For the first time, we found 30 cases of coexistence of EGFR and KRAS mutations in FFPE samples of NSCLCs. In other words, a tumour can simultaneously harbour an activating EGFR mutation as well as mutations downstream of the pathway in the KRAS gene, which means that upstream inhibition of EGFR with TKIs will have no therapeutic effect in these cases. This finding also suggests that the EGFR mutation test alone may not be sufficient for the decision of whether or not the patient is a candidate for TKI therapy.

Interestingly, our data showed that KRAS and BRAF V600E mutations are mutually exclusive, which agrees with Rajagopalan et al (2002) that the BRAF mutation occurs only in tumours that do not carry KRAS mutations, as KRAS and BRAF provide an equivalent or at least redundant oncogenic stimulus in cancer pathogenesis. This phenomenon has also been observed in colorectal cancer (De Roock et al, 2010). However, we did find for the first time two cases of EGFR and BRAF coexsiting mutations, which have been previously considered as mutually exclusive mutations, as well. Again, we submit that KRAS and $B R A F$ mutation tests should be run concurrently with the EGFR mutation test.

EGFR-TKIs, such as gefitinib and erlotinib, have been successfully used to treat advanced NSCLCs with EGFR-activating mutations, exon 19 deletions or L858R. However, over 6-12 months of treatment, most tumours acquire resistance to EGFR-TKIs. Furthermore, it was discovered that the secondary T790M mutation occurs in $50 \%$ of EGFR-mutated patients with TKI resistance (Oxnard et al, 2011; Ohashi et al, 2012). Both EGFR T790M and PIK3CA E545K are considered as acquired resistance mutations to TKI treatment (Engelman et al, 2006; Engelman and Janne, 2008), and are considered as biomarkers for poor clinical prognosis. Among our 160 cases of multiple mutations, an EGFR-activating mutation plus a T790M point mutation was accounted for in 32 cases $(20 \%)$, and 24 cases (15\%) also included E545K. Twelve of them $(12, \mathrm{~T} 790 \mathrm{M}$ and $0, \mathrm{E} 545 \mathrm{~K})$ might be the results of TKI therapy. However, it was very interesting to find that there were 44 cases (20, T790M and 24, E545K) where patients had never received any TKI therapy but already harboured T790M or E545K secondary mutations. This may explain why some NSCLCs who harbour EGFR-activating mutations still fail the TKI therapy (Kobayashi et al, 2005). The same double mutations have been previously described in two patients who showed very poor responses to TKI treatment (Toyooka et al, 2005). Moreover, data have shown that continued activation of PI3K signalling by the PIK3CA oncogenic mutant, p110 $\alpha$ E545K, is sufficient to abrogate gefitinib-induced apoptosis (Subramanian et al, 2010). Together, our data confirmed the coexistence of the EGFR-activating mutation of exon 19 deletions or L858R, and the resistant point mutation of T790M or E545K before the TKI treatment. This finding provides evidence that double mutations with a second resistant mutation are important factors conferring resistance to TKI therapy in NSCLC carrying EGFR-sensitive mutations, thus lending insight to further considerations for designing clinical trials, or drugs, for more effective strategies against specific subtypes of NSCLC with both EGFR-TKIs and anti-T790M therapies. In the future, a more sensitive technology 
should be able to identify whether these mutations are in the same cellule or not.

Together, our data present a clear panoramagram of mutation frequencies of EGFR, KRAS, BRAF and PIK3CA from 5125 NSCLC cases and their associations with gender, smoking history and histology. For the first time, 153 double and seven triple mutation cases were identified in NSCLC. These data will lead further investigations to explore the associations between multiple mutations and clinical outcomes. Finally, we also reported here, for the first time, the detection of KRAS mutations in children with NSCLC.

\section{ACKNOWLEDGEMENTS}

We wish to thank Zhiping Cai, Xiaoxia Qin, Huijuan Qin, Xuqiu Qin, Keyin Luo, Zhangmin Liang, Xinsheng Li, Yuting $\mathrm{Yu}$ and Qingpeng Zhong for their technical assistance. This research was supported by the Subtask of National Science and Technology Major Projects of China 'Key New Drug Creation and Manufacturing Program' (grant number 2012ZX09506001-003); by a grant from the Chinese Ministry of Education, the Key Project Grant (grant number 311037; PI: SL); and by the Young Investigator Award from Peking Union Medical College Hospital (grant number 2010116, PI: NL).

\section{CONFLICT OF INTEREST}

The authors declare no conflict of interest.

\section{REFERENCES}

Age Group of Lung Cancer Diagnosed (2000-2007) http://www.aboutcancer. com/lunglb.htm.

American Cancer Society (2011) Cancer Facts and Figures 2009. http://ww2. cancer.org/downloads/STT/500809web.pdf.

Asahina H, Yamazaki K, Kinoshita I, Yokouchi H, Dosaka-Akita H, Nishimura M (2006) Non-responsiveness to gefitinib in a patient with lung adenocarcinoma having rare EGFR mutations S768I and V769L. Lung Cancer 54(3): 419-422.

Besaratinia A, Pfeifer GP (2008) Second-hand smoke and human lung cancer. Lancet Oncol 9(7): 657-666.

Chaft JE, Arcila ME, Paik PK, Lau C, Riely GJ, Pietanza MC, Zakowski MF, Rusch V, Sima CS, Ladanyi M, Kris MG (2012) Coexistence of PIK3CA and other oncogene mutations in lung adenocarcinoma-rationale for comprehensive mutation profiling. Mol Cancer Ther 11(2): 485-491.

D'Angelo SP, Pietanza MC, Johnson ML, Riely GJ, Miller VA, Sima CS, Zakowski MF, Rusch VW, Ladanyi M, Kris MG (2011) Incidence of EGFR exon 19 deletions and L858R in tumor specimens from men and cigarette smokers with lung adenocarcinomas. J Clin Oncol 29(15): 2066-2070.

De Roock W, Claes B, Bernasconi D, De Schutter J, Biesmans B, Fountzilas G, Kalogeras KT, Kotoula V, Papamichael D, Laurent-Puig P, Penault-Llorca F, Rougier P, Vincenzi B, Santini D, Tonini G, Cappuzzo F, Frattini M, Molinari F, Saletti P, De Dosso S, Martini M, Bardelli A, Siena S, Sartore-Bianchi A, Tabernero J, Macarulla T, Di Fiore F, Gangloff AO, Ciardiello F, Pfeiffer P, Qvortrup C, Hansen TP, Van Cutsem E, Piessevaux H, Lambrechts D, Delorenzi M, Tejpar S (2010) Effects of KRAS, BRAF, NRAS, and PIK3CA mutations on the efficacy of cetuximab plus chemotherapy in chemotherapy-refractory metastatic colorectal cancer: a retrospective consortium analysis. Lancet Oncol 11(8): 753-762.

Dogan S, Shen R, Ang DC, Johnson ML, D’Angelo SP, Paik PK, Brzostowski EB, Riely GJ, Kris MG, Zakowski MF, Ladanyi M (2012) Molecular epidemiology of EGFR and KRAS mutations in 3026 lung adenocarcinomas: higher susceptibility of women to smoking-related KRAS-mutant cancers. Clin Cancer Res 18(22): 6169-6177.

Engelman JA, Janne PA (2008) Mechanisms of acquired resistance to epidermal growth factor receptor tyrosine kinase inhibitors in non-small cell lung cancer. Clin Cancer Res 14(10): 2895-2899.
Engelman JA, Mukohara T, Zejnullahu K, Lifshits E, Borrás AM, Gale CM, Naumov GN, Yeap BY, Jarrell E, Sun J, Tracy S, Zhao X, Heymach JV, Johnson BE, Cantley LC, Jänne PA (2006) Allelic dilution obscures detection of a biologically significant resistance mutation in EGFRamplified lung cancer. J Clin Invest 116(10): 2695-2706.

Jemal A, Bray F, Center MM, Ferlay J, Ward E, Forman D (2011) Global cancer statistics. CA Cancer J Clin 61(2): 69-90.

Kobayashi S, Boggon TJ, Dayaram T, Jänne PA, Kocher O, Meyerson M, Johnson BE, Eck MJ, Tenen DG, Halmos B (2005) EGFR mutation and resistance of non-small-cell lung cancer to gefitinib. N Engl J Med 352(8): 786-792.

Li G, Luo X, He J, Zhu Z, Yu G, Qin H, Zeng T, Liu Z, Wu S, Xu J, Ren-Heidenreich L (2011) A novel liquidchip platform for simultaneous detection of 70 alleles of DNA somatic mutations on EGFR, KRAS, BRAF and PIK3CA from formalin-fixed and paraffin-embedded slides containing tumor tissue. Clin Chem Lab Med 49(2): 191-195.

Lin L, Bivona TG (2012) Mechanisms of resistance to epidermal growth factor receptor inhibitors and novel therapeutic strategies to overcome resistance in NSCLC patients. Chemother Res Pract 2012: 817297.

Ludovini V, Bianconi F, Pistola L, Chiari R, Minotti V, Colella R, Giuffrida D, Tofanetti FR, Siggillino A, Flacco A, Baldelli E, Iacono D, Mameli MG, Cavaliere A, Crinò L (2011) Phosphoinositide-3-kinase catalytic alpha and KRAS mutations are important predictors of resistance to therapy with epidermal growth factor receptor tyrosine kinase inhibitors in patients with advanced non-small cell lung cancer. J Thorac Oncol 6(4): 707-715.

Lynch TJ, Bell DW, Sordella R, Gurubhagavatula S, Okimoto RA, Brannigan BW, Harris PL, Haserlat SM, Supko JG, Haluska FG, Louis DN, Christiani DC Settleman J, Haber DA (2004) Activating mutations in the epidermal growth factor receptor underlying responsiveness of non-small-cell lung cancer to gefitinib. N Engl J Med 350(21): 2129-2139.

Marchetti A, Felicioni L, Malatesta S, Grazia Sciarrotta M, Guetti L, Chella A, Viola P, Pullara C, Mucilli F, Buttitta F (2011) Clinical features and outcome of patients with non-small-cell lung cancer harboring BRAF mutations. J Clin Oncol 29(26): 3574-3579.

Marchetti A, Martella C, Felicioni L, Barassi F, Salvatore S, Chella A, Camplese PP, Iarussi T, Mucilli F, Mezzetti A, Cuccurullo F, Sacco R, Buttitta F (2005) EGFR mutations in non-small-cell lung cancer: analysis of a large series of cases and development of a rapid and sensitive method for diagnostic screening with potential implications on pharmacologic treatment. J Clin Oncol 23(4): 857-865.

Masago K, Fujita S, Irisa K, Kim YH, Ichikawa M, Mio T, Mishima M (2010) Good clinical response to gefitinib in a non-small cell lung cancer patient harboring a rare somatic epidermal growth factor gene point mutation; codon 768 AGC > ATC in exon 20 (S768I). Jpn J Clin Oncol 40(11): 1105-1109.

Ohashi K, Sequist LV, Arcila ME, Moran T, Chmielecki J, Lin YL, Pan Y, Wang L, de Stanchina E, Shien K, Aoe K, Toyooka S, Kiura K, Fernandez-Cuesta L, Fidias P, Yang JC, Miller VA, Riely GJ, Kris MG, Engelman JA, Vnencak-Jones CL, Dias-Santagata D, Ladanyi M, Pao W (2012) Lung cancers with acquired resistance to EGFR inhibitors occasionally harbor BRAF gene mutations but lack mutations in KRAS, NRAS, or MEK1. Proc Natl Acad Sci USA 109(31): E2127-E2133.

Oxnard GR, Arcila ME, Chmielecki J, Ladanyi M, Miller VA, Pao W (2011) New strategies in overcoming acquired resistance to epidermal growth factor receptor tyrosine kinase inhibitors in lung cancer. Clin Cancer Res 17(17): 5530-5537.

Paik PK, Arcila ME, Fara M, Sima CS, Miller VA, Kris MG, Ladanyi M, Riely GJ (2011) Clinical characteristics of patients with lung adenocarcinomas harboring BRAF mutations. J Clin Oncol 29(15): 2046-2051.

Pao W, Miller V, Zakowski M, Doherty J, Politi K, Sarkaria I, Singh B, Heelan R, Rusch V, Fulton L, Mardis E, Kupfer D, Wilson R, Kris M, Varmus H (2004) EGF receptor gene mutations are common in lung cancers from "never smokers" and are associated with sensitivity of tumors to gefitinib and erlotinib. Proc Natl Acad Sci USA 101(36): 13306-13311.

Pao W, Wang TY, Riely GJ, Miller VA, Pan Q, Ladanyi M, Zakowski MF, Heelan RT, Kris MG, Varmus HE (2005) KRAS mutations and primary resistance of lung adenocarcinomas to gefitinib or erlotinib. PLoS Med 2(1): e17.

Rajagopalan H, Bardelli A, Lengauer C, Kinzler KW, Vogelstein B, Velculescu VE (2002) Tumorigenesis: RAF/RAS oncogenes and mismatch-repair status. Nature 418(6901): 934.

Riely GJ, Kris MG, Rosenbaum D, Marks J, Li A, Chitale DA, Nafa K, Riedel ER, Hsu M, Pao W, Miller VA, Ladanyi M (2008) Frequency and distinctive 
spectrum of KRAS mutations in never smokers with lung adenocarcinoma. Clin Cancer Res 14(18): 5731-5734.

Rodenhuis S, van de Wetering ML, Mooi WJ, Evers SG, van Zandwijk N, Bos JL (1987) Mutational activation of the K-ras oncogene. A possible pathogenetic factor in adenocarcinoma of the lung. N Engl J Med 317(15): 929-935.

Rosell R, Moran T, Queralt C, Porta R, Cardenal F, Camps C, Majem M, Lopez-Vivanco G, Isla D, Provencio M, Insa A, Massuti B,

Gonzalez-Larriba JL, Paz-Ares L, Bover I, Garcia-Campelo R, Moreno MA, Catot S, Rolfo C, Reguart N, Palmero R, Sánchez JM, Bastus R, Mayo C, Bertran-Alamillo J, Molina MA, Sanchez JJ, Taron M. Spanish Lung Cancer Group (2009) Screening for epidermal growth factor receptor mutations in lung cancer. N Engl J Med 361(10): 958-967.

Sasaki H, Kawano O, Endo K, Suzuki E, Haneda H, Yukiue H, Kobayashi Y, Yano M, Fujii Y (2006) Uncommon V599E BRAF mutations in Japanese patients with lung cancer. J Surg Res 133(2): 203-206.

Schmid K, Oehl N, Wrba F, Pirker R, Pirker C, Filipits M (2009) EGFR/KRAS/ BRAF mutations in primary lung adenocarcinomas and corresponding locoregional lymph node metastases. Clin Cancer Res 15(14): 4554-4560.

Slebos RJ, Kibbelaar RE, Dalesio O, Kooistra A, Stam J, Meijer CJ, Wagenaar SS, Vanderschueren RG, van Zandwijk N, Mooi WJ, Bos JL, Rodenhuis S (1990) K-ras oncogene activation as a prognostic marker in adenocarcinoma of the lung. $N$ Engl J Med 323(9): 561-565.

Smits AJ, Kummer JA, Hinrichs JW, Herder GJ, Scheidel-Jacobse KC, Jiwa NM, Ruijter TE, Nooijen PT, Looijen-Salamon MG, Ligtenberg MJ, Thunnissen FB, Heideman DA, de Weger RA, Vink A (2012) EGFR and KRAS mutations in lung carcinomas in the Dutch population: increased
EGFR mutation frequency in malignant pleural effusion of lung adenocarcinoma. Cell Oncol (Dordr) 35(3): 189-196.

Subramanian J, Morgensztern D, Goodgame B, Baggstrom MQ, Gao F, Piccirillo J, Govindan R (2010) Distinctive characteristics of non-small cell lung cancer (NSCLC) in the young: a surveillance, epidemiology, and end results (SEER) analysis. J Thorac Oncol 5(1): 23-28.

Toyooka S, Kiura K, Mitsudomi T (2005) EGFR mutation and response of lung cancer to gefitinib. N Engl J Med 352(20): 2136.

Wu S, Zhu Z, He J, Luo X, Xu J, Ren-Heidenreich L (2010) A novel mutant-enriched liquidchip technology for the qualitative detection of somatic mutations in KRAS gene from both serum and tissue samples. Clin Chem Lab Med 48(8): 1103-1106.

Xu J, He J, Yang H, Luo X, Liang Z, Chen J, Cai Z, Xu J, Ren-Heidenreich L (2011) Somatic mutation analysis of EGFR, KRAS, BRAF and PIK3CA in 861 patients with non-small cell lung cancer. Cancer Biomark 10(2): 63-69.

Zhang L, Yang H, Zhao Y, Liu W, Wu S, He J, Luo X, Zhu Z, Xu J, Zhou Q, Ren-Heidenreich L (2012) Detection of EGFR somatic mutations in non-small cell lung cancer (NSCLC) using a novel mutant-enriched liquidchip (MEL) technology. Curr Drug Metab 13(7): 1007-1011.

This work is published under the standard license to publish agreement. After 12 months the work will become freely available and the license terms will switch to a Creative Commons AttributionNonCommercial-Share Alike 3.0 Unported License. 